\title{
Counterrotating Bars
}

\author{
Chad L. Davies \\ Department of Physics, University of Florida, Gainesville, FL 32611 \\ James H. Hunter, Jr. \\ Department of Astronomy, University of Florida, P.O. Box 112055, \\ Gainesville, FL 32611-2055
}

\begin{abstract}
By including counterrotating angular momentum in twodimensional galactic disk simulations, we are able to create counterrotating bar modes. Over the period of several dynamical times, the counterrotating inner bar is shown to reverse its pattern direction, oscillate around the primary bar and finally align with it.
\end{abstract}

\section{Introduction}

With the recent observations of several disk galaxies containing significant portions of counterrotating (CR) angular momentum (NGC 4550: Rubin et al. 1992, Rix et al. 1992; NGC 7217: Merrifield \& Kuijken 1994; NGC 4826: Braun et al. 1994, Walterbos et al. 1994, Rix et al. 1994, Rubin 1994; NGC 7332: Fisher et al. 1994; NGC 4546: Galletta 1987, Bettoni et al. 1991, Sage \& Galletta 1994), the question arises as to what dynamical effects will such CR components have on the global evolution of disk systems. With this in mind, we have undertaken a modeling program that examines two dimensional disk systems with initial conditions that include CR angular momentum.

\section{Initial Conditions}

The initial conditions used for the disks to be investigated are taken from the consistently truncated Toomre family (Toomre 1963, Hunter, Ball \& Gottesman 1984). We are able to parametrize the ordered and dispersional motions of the disk using a collisionless Boltzmann equation (CBE) formalism that allows us to specify the Ostriker-Peebles (OP) (1973) parameter for each disk. We include $\mathrm{CR}$ angular momentum in the initial conditions in one of two ways. In the first case, which we refer to as fully mixed (FM), we reverse a percentage of the angular momentum throughout the disk. In the second case, which we refer to as the step function (STF) case, we reverse all of the angular momentum of the disk inside a critical radius. A sub-case of this is the slope function (SLF) case, wherein the percentage of CR angular momentum is varied linearly in an annular ring inside of which all the angular momentum is retrograde and outside of which all of the orbits are direct. These initial conditions are evolved using a 
hierarchical tree algorithm kindly provided to us by C. Heller and I. Shlosman. We have chosen machine units of length and mass such that the dynamical time of the system is 47.1 million years.

\section{Simulation Results}

\subsection{Fully Mixed (FM) Cases}

For the FM cases we have run, we find that a disk constructed to be bar unstable according to the OP parameter will be stabilized by a sufficient amount of CR angular momentum. For a Toomre $\mathrm{n}=0$ disk we find the amount necessary to be approximately $15 \%$, while for Toomre $n=1$ disks this amount is approximately $25 \%$. We are able to avoid the $\mathrm{m}=1$ instability reported by a number of other authors (Kalnajs 1976, Zang \& Hohl 1978, Araki 1987) by choosing our ratio of ordered azimuthal rotation to cold rotation below Araki's critical value of 0.7071 .

\subsection{Step/Slope Function (STF/SLF) Cases}

Toomre $n=0$ disks Using STF initial conditions for a Toomre $\mathrm{n}=0$ disk, we have found that models with sufficient $\mathrm{CR}$ angular momentum evolve rather differently than fully direct cases. In the Toomre $n=0$ disk, it is well known that models constructed to be bar unstable will evolve to a large, slowly rotating bar with little remaining disk. We find that if we reverse approximately $50 \%$ of the angular momentum in a STF fashion, the system evolves towards an equilibrium configuration which has both a disk and a CR truncated bar that rotates with approximately $1 / 3$ of the pattern speed of the bar in the fully direct case.

Toomre $n=1$ disks Similar to the Toomre $n=0$ case previously discussed, we are able to construct models that evolve to bar/disk configurations where the bar counterrotates with fractional pattern speed. However, we find that if we construct the initial conditions to have only $10 \%$ of the angular momentum in retrograde orbits, we find that the evolution is quite different. In these models, we see the development of a short, rapidly rotating, inner $\mathrm{CR}$ bar. On a longer time scale, a larger, directly rotating bar forms. As the simulation progresses, the inner bar slows, reverses its pattern direction and then oscillates with progressively smaller amplitude around the larger bar. For $\mathrm{N}=32000$, we find that this process takes place in approximately 1.9 Gyrs. If $10 \%$ of the angular momentum is distributed by using the SLF model, we are able to develop a long lived, axisymmetrically stable core and a directly rotating bar.

\section{Analysis and Conclusions}

In this study we have found the following results:

1. We are able to stabilize disks that are, when constructed with fully direct angular momentum, unstable to the formation of $m=2$ modes.

2. We are able to produce systems that consist of inner and outer bars that counterrotate with respect to each other. Observations by Friedli and 
Wozniak (Wozniak et al. 1995, Friedli 1995, Friedli et al. 1995) have revealed that systems with multiple bar configurations do, in fact, exist; though it is unknown whether any of these systems display counterrotation.

3. We are able to produce bar/disk configurations with slowly rotating bars in disks with rising rotation curves. Also, we find that it is possible to construct systems that result in bar/bulgelike configurations.

While these simulations do not confirm the existence of such types of galaxies, they do show that such configurations are possible to construct. Though the question of how such systems come about is still unanswered, likely mechanisms include galaxy/galaxy interactions, bar destruction and torques due to distant neighbors during the formation process (Voglis et al. 1991).

Acknowledgments. We wish to thank G. Contopoulos, I. Shlosman, D. Christodoulou and D. Friedli for a number of insightful discussions. This research was supported in part by NSF grant AST9022827, the University of Florida Division of Sponsored Research and the Research Computing Initiative, a collaboration of the University of Florida and International Business Machines through the Northeast Regional Data Center.

\section{References}

Araki, S. 1987, AJ, 94, 99

Bettoni, D., Galletta G., \& Oosterloo, T. 1991, MNRAS, 248, 544

Braun, R., Walterbos, R. A. M., Kennicutt, R. C., Jr., \& Tacconi, L. J. 1994, ApJ, 420, 558

Fisher, D., Illingworth, G., \& Franx, M. 1994, AJ, 107, 160

Galletta, G. 1987, ApJ, 318, 531

Hunter, J. H., Jr., Ball, R., \& Gottesman, S. T. 1984, MNRAS, 208, 1

Kalnajs, A. J. 1976, ApJ, 205, 751

Merrifield, M. R. \& Kuijken, K. 1994, ApJ, 432, 575

Ostriker, J. P. \& Peebles, P. J. E. 1973, ApJ, 186, 467

Rix, H.-W., Franx, M., Fisher, D., \& Illingworth, G. 1992, ApJ, 400, L5

Rix, H.-W., Kennicutt, R. C., Jr., Braun, R., \& Walterbos, R. A. M. 1995, ApJ, 438,155

Rubin, V. C. 1994, AJ, 107, 173

Rubin, V. C., Graham, J., \& Kenney, J. D. P. 1992, ApJ, 394, L9

Sage, L. \& Galletta, G. 1994, AJ, 108, 1633

Toomre, A. 1963, ApJ, 138, 385

Voglis, N., Hiotelis, N., \& Holflich, D. 1991, A\&A, 248, 5

Walterbos, R. A. M., Braun, R., \& Kennicutt, R. C., Jr. 1994, 107, 184

Wozniak, H., Friedli, D., Martinet, L., Martin, P., \& Bratschi, P. 1995, A\&AS, 111,115

Zang, T. A. \& Hohl, F. 1978, ApJ, 226, 521 